\title{
Impact of phthalate and BPA exposure during in utero windows of susceptibility on reproductive hormones and sexual maturation in peripubertal males
}

Deborah J. Watkins ${ }^{1 *}$, Brisa N. Sánchez², Martha Maria Téllez-Rojo ${ }^{3}$, Joyce M. Lee ${ }^{1,4}$, Adriana Mercado-García ${ }^{3}$, Clara Blank-Goldenberg ${ }^{5}$, Karen E. Peterson ${ }^{6,7}$ and John D. Meeker ${ }^{1}$

\begin{abstract}
Background: Phthalates and BPA are endocrine disrupting chemicals (EDCs) widely used in consumer products. Evidence suggests that phthalate and BPA exposure alters steroid hormone levels in adults, while in utero exposure has been associated with altered fetal reproductive development in boys. However, the impact of exposure during distinct critical windows of in utero development on hormone concentrations and sexual maturation during the pubertal transition has not been examined. The objective of this study was to assess trimester-specific in utero phthalate and BPA exposure in relation to measures of reproductive development among peripubertal boys in a Mexico City birth cohort.

Methods: We measured maternal urinary phthalate metabolites and BPA during the first, second, and third trimesters of pregnancy. We measured serum levels of testosterone, estradiol, dehydroepiandrosterone sulfate (DHEA-S), inhibin B, and sex hormone-binding globulin (SHBG), and assessed sexual maturation (Tanner staging and testicular volume) among male children at age 8-14 years $(n=109)$. Linear and logistic regression were used to investigate trimester-specific in utero exposure as predictors of peripubertal hormone levels and sexual maturation, respectively. In sensitivity analyses we evaluated estimated exposure at 7 weeks gestation and rates of change in exposure across pregnancy in relation to outcomes.

Results: Exposure to phthalates during the third trimester was associated with reduced odds of having a Tanner stage $>1$ for pubic hair development (e.g. MBzP OR $=0.18$ per interquartile range (IQR) increase; 95\% Cl:0.03-0.97) and higher peripubertal SHBG levels (e.g. MBzP 15.2\%/IQR; 95\% Cl:3.2-28\%), while first and second trimester phthalates were not. In contrast, exposure to DEHP during the first trimester was associated with higher estradiol (11\%/IQR; 95\% Cl:1.5-22\%), while second or third trimester DEHP exposure was not. Sensitivity analyses yielded similar findings.

Conclusions: Associations between in utero phthalate and BPA exposure and peripubertal measures of male reproductive development are dependent on the timing of that exposure during gestation. These findings suggest that future epidemiological studies relating in utero EDC exposure to pubertal outcomes should consider windows of susceptibility.
\end{abstract}

Keywords: In utero exposure, Windows of susceptibility, Puberty, Phthalates, BPA

\footnotetext{
* Correspondence: debjwat@umich.edu

'Department of Environmental Health Sciences, University of Michigan

School of Public Health, 6611C SPH I, 1415 Washington Heights, Ann Arbor,

Ml 48109, USA

Full list of author information is available at the end of the article
} 


\section{Background}

Phthalates and bisphenol A (BPA) are endocrine disrupting chemicals (EDCs) [1] that are widely used in a range of consumer products, resulting in ubiquitous human exposure [2-4]. Evidence suggests that exposure to phthalates and BPA alters reproductive hormone levels in adults [5-9], while exposure during in utero development has been associated with changes in reproductive hormone levels in newborns $[10,11]$. In addition, several studies have reported associations between in utero phthalate exposure and shortened anogenital distance (AGD) [12-14], a marker of decreased androgen exposure during in utero reproductive development and a potential indicator of reduced male fertility in adulthood $[15,16]$. However, the impact of in utero phthalate and BPA exposure on peripubertal reproductive hormone concentrations and sexual maturation is unclear.

In earlier work, we reported that phthalate exposure during the third trimester of in utero development was associated with lower odds of adrenarche, while peripubertal exposure was not [17]. Given that androgen action during relatively short, specific fetal periods is necessary for male reproductive development [18, 19], the objective of the present analysis was to utilize newly available data to assess early in utero windows of susceptibility to phthalate and BPA exposure among boys in this study population. To our knowledge, this is the first study to evaluate maternal urinary phthalate metabolite and BPA concentrations during their first, second, and third trimesters of pregnancy, as well as the level and rate of change of exposure across pregnancy, in relation to peripubertal reproductive hormone levels and measures of sexual maturation among their male children.

\section{Methods}

\section{Study population}

Participants are part of the Early Life Exposure in Mexico to Environmental Toxicants (ELEMENT) project, a longitudinal cohort study of pregnant women in Mexico City and their offspring. Our analysis includes women who were recruited from maternity hospitals during their first trimester between 1997 and 2004 and their offspring as previously described [20]. Mothers provided a urine sample and completed interview-based questionnaires at up to three different prenatal visits (mean gestational age at visit 1: 13.5 weeks [range 5-24], visit 2: 25.1 weeks [range 17-37], and visit 3: 34.4 weeks [range 26-43]). Gestational age was based on the selfreported date of each mother's last menstrual period. In 2010, a subset of their children, who were then 8 to 14 years of age, were re-contacted to participate in follow-up studies $(n=250)$. Participants were selected from the overall ELEMENT study based on the availability of archived prenatal urine samples for biomarker measurement. Children provided serum samples, anthropometry, and demographic information via an interview-based questionnaire. Age-specific body mass index (BMI) zscores were calculated based on the World Health Organization growth standards for age and sex [21]. In the current analyses, we included male children for whom we had maternal urinary phthalate metabolite and BPA measurements from at least one prenatal study visit $(n=109)$. Research protocols were approved by the ethics and research committees of the Mexico National Institute of Public Health and the University of Michigan, and all participants provided informed consent prior to enrollment.

\section{Urinary phthalate metabolites and BPA}

Each mother provided a second morning void urine sample during at least one of the three prenatal study visits (visit $1 n=92$, visit $2 n=91$, visit $3 n=108$; $n=85$ provided a sample at all three time points). Samples were frozen and stored at $-80{ }^{\circ} \mathrm{C}$ at the University of Michigan until analysis at NSF International (Ann Arbor, MI). BPA and nine phthalate metabolites, including monoethyl phthalate (MEP), mono- $n$-butyl phthalate (MnBP), monoisobutyl phthalate (MiBP), monobenzyl phthalate (MBzP), mono-3-carboxypropyl phthalate (MCPP), mono-2-ethylhexyl phthalate (MEHP), mono-2-ethyl-5-hydroxyhexyl phthalate (MEHHP), mono-2-ethyl-5-oxohexyl phthalate (MEOHP), and mono-2-ethyl-5-carboxypentyl phthalate (MECPP) were measured using isotope dilution-liquid chromatographytandem mass spectrometry (ID-LC-MS/MS) as previously described [20]. We calculated a di-2-ethylhexyl phthalate (DEHP) metabolite summary measure ( $\Sigma \mathrm{DEHP})$ for each sample by dividing individual MEHP, MEHHP, MEOHP, and MECPP concentrations by their molar mass and summing them. We calculated a summary dibutyl phthalate (DBP) metabolite measure ( $\mathrm{DDBP})$ as the molar sum of MnBP and MiBP. Specific gravity (SG) was measured using a handheld digital refractometer (Atago Co., Ltd., Tokyo, Japan) at the time of sample analysis. For select analyses only, phthalate metabolite and BPA values were corrected for urinary SG using the following equation: $P_{c}=P\left[\left(S G_{p}-1\right) /\left(S_{i}-1\right)\right]$ where $P_{c}$ is the SGcorrected phthalate metabolite or BPA concentration (ng/ $\mathrm{mL}$ ), $\mathrm{P}$ is the measured phthalate metabolite or BPA concentration, $\mathrm{SG}_{\mathrm{p}}$ is the median urinary specific gravity, and $\mathrm{SG}_{\mathrm{i}}$ is the individual's urinary specific gravity. Spearman correlations between trimester specific measures of SG, phthalate metabolites, and BPA are presented in (Additional file 1: Table S1). Values below the limit of detection (LOD) were replaced with the LOD $/ \sqrt{ } 2$. 


\section{Hormones}

Children provided fasting blood samples during the follow-up visit at age 8-14 years. Serum aliquots were separated and frozen at $-80{ }^{\circ} \mathrm{C}$, and then sent to the Clinical Ligand Assay Service Satellite (CLASS) Laboratory at the University of Michigan (Ann Arbor, MI) for hormone analysis. We measured estradiol, testosterone, inhibin $\mathrm{B}$, and sex hormone-binding globulin (SHBG) as biomarkers of puberty, and dehydroepiandrosterone sulfate (DHEA-S) as a biomarker of adrenarche. Estradiol, total testosterone, SHBG, and DHEA-S were measured using an automated chemiluminescent immunoassay (Bayer Diagnostics ACS:180). Active inhibin B was assayed using Gen II ELISA (Beckman Coulter, Webster, TX). Values below the limit of detection (LOD) were replaced with the LOD $/ \sqrt{ } 2$.

\section{Sexual maturation}

A trained pediatrician evaluated male offspring for Tanner staging and testicular volume using standardized protocols. We used genital stage (GD) as an indicator of puberty and pubic hair stage $(\mathrm{PH})$ as an indicator of adrenarche, with stage 1 corresponding to no development and stage 5 corresponding to full development [22]. For analyses, Tanner stages were converted to dichotomous variables with pubertal onset defined by having a GD stage $>1$ and adrenarche defined by having a $\mathrm{PH}$ stage $>1$. Right and left testicular volume (TV) were measured with an orchidometer, and the larger of the two measurements was used in analyses. As a testicular volume of $1-3 \mathrm{~mL}$ is considered prepubertal, a cutoff of $3 \mathrm{~mL}$ was used to denote no puberty $(\leq 3 \mathrm{~mL})$ vs. puberty (>3 mL) [23].

\section{Statistical methods}

Serum hormone and urinary phthalate metabolite and BPA concentrations were log-normally distributed, and ln-transformed prior to analysis. We used mixed models to calculate intraclass correlation coefficients (ICCs) and 95\% confidence intervals to examine the variability of phthalate and BPA measurements across pregnancy within individuals with more than one exposure measurement [24]. Linear regression was used to separately assess associations between visit-specific phthalate metabolite and BPA concentrations and peripubertal serum hormone concentrations, adjusting a priori for child age and BMI Z-score, and for SG as a measure of urinary dilution. To assess overall exposure during pregnancy, we calculated geometric mean (GM) phthalate metabolite and BPA concentrations for each individual using all available measurements from prenatal visits 1,2 , and 3 . If only one measurement from one prenatal visit was available, that value was used as the GM. Individual GM values were then entered into regression models to assess relationships between overall in utero exposure and peripubertal hormone levels. Results are presented as the percent change in hormone (95\% confidence interval) per interquartile range (IQR) increase in phthalate metabolite or BPA. All results are calculated based on the IQR of GM concentrations across pregnancy in order to more easily compare visit-specific effect estimates.

We used logistic regression to separately examine associations between visit-specific urinary phthalate metabolite or BPA concentrations and the odds of pubertal onset or adrenarche using dichotomous measures of sexual maturation (GD, PH, TV). In full models, child age and BMI z-score were included as covariates a priori. As few participants were categorized as having a $\mathrm{PH}$ Tanner stage $>1$ (Additional file 1: Table S2), the number of covariates we could reliably enter into models was limited $[25,26]$. Thus, to minimize the number of covariates in logistic models, we included SG-corrected phthalate metabolite and BPA concentrations rather than entering SG as a separate covariate. Individual GM phthalate metabolite and BPA values were also entered into logistic regression models to assess relationships of overall in utero exposure with odds of pubertal onset and adrenarche. Results are presented as odds ratios (OR, 95\% confidence interval), which express the change in odds per IQR increase in exposure, with the IQR based on GM concentrations across pregnancy.

We carried out complementary, sensitivity analyses for each exposure that utilized data from all participants and all visits in a single model to examine whether the level (intercept) or rate of change (slope) of the exposure during pregnancy was associated with hormone concentrations or sexual maturation. To carry out this analysis, we first used mixed effects models with gestational age as a predictor of ln-transformed, SG-corrected phthalate or BPA concentrations to estimate, for each woman and each compound, a random intercept and slope that described the compound's trajectory during the course of pregnancy. Prior to entering gestational age into the model, it was centered at 7 weeks, such that an individual's intercept represents urinary phthalate metabolite or BPA concentrations at 7 weeks, the mid-point of the first trimester of pregnancy. An individual's slope indicates the rate of change in urinary phthalate metabolite or BPA across pregnancy. For each exposure, we then entered the estimated slope and intercept variables into regression models as predictors of either hormone concentrations or measures of sexual maturation. However, if the slope and intercept for a specific phthalate metabolite or BPA were highly correlated (Pearson $r>0.8$ ), or if effect estimates were imprecise (e.g. infinitely wide $95 \%$ confidence intervals), only the intercept was used. Findings were considered statistically significant at $p<0.05$, and all analyses were performed using SAS version 9.3 (Cary, NC). 


\section{Results}

The percent of samples with phthalate metabolite or BPA concentrations below the limit of detection, as well as the geometric means and standard deviations of sample concentrations from prenatal visits 1,2 , and 3 are shown in Table 1. Urinary BPA concentrations significantly decreased, while MBzP, MEOHP, and MiBP concentrations significantly increased across pregnancy. ICCs for phthalate metabolite and BPA concentrations among all mothers ranged from 0.21 for MBzP to 0.41 for MEP, suggesting substantial within individual variability. Differences in levels across pregnancy and the percent of samples below the LOD were similar when we limited our analysis to only mothers who had male children, although ICCs ranged from 0.14 for $\Sigma$ DEHP to 0.47 for MEP. The distribution of serum hormone levels and measures of sexual maturation among this population have been previously reported elsewhere [17].

Maternal phthalate metabolite and BPA concentrations at prenatal visit 1 were not associated with peripubertal hormone levels in male offspring at 8-14 years of age, with the exception of $\Sigma D E H P$, for which an IQR increase was associated with 11\% (95\% CI: 1.5, 21.9) higher serum estradiol after adjustment for child age, BMI zscore, and urinary SG (Table 2). At prenatal visit 2, an IQR increase in MEP was associated with 15\% (95\% CI: -25.1, -3.6) lower estradiol concentrations, while an IQR increase in MBzP was associated with $16 \%$ (95\% CI: -26.8, -3.4) lower DHEA-S. Maternal BPA concentrations at prenatal visit 2 were also associated

Table 1 Distribution of urinary phthalate metabolites and BPA across pregnancy $(\mu \mathrm{g} / \mathrm{L})$

\begin{tabular}{|c|c|c|c|c|c|c|c|c|c|c|c|c|c|c|c|c|}
\hline \multirow[b]{2}{*}{ All } & \multirow[b]{2}{*}{$\%<\mathrm{LOD}^{\mathrm{a}}$} & \multicolumn{3}{|c|}{ Visit $1(n=199)$} & \multicolumn{3}{|c|}{ Visit $2(n=200)$} & \multicolumn{3}{|c|}{ Visit $3(n=225)$} & \multicolumn{3}{|c|}{ Prenatal GM $(n=229)$} & \multicolumn{3}{|c|}{ Variability } \\
\hline & & \multicolumn{2}{|c|}{ GM (GSD) } & \multirow{2}{*}{$\begin{array}{l}\text { Max } \\
9.3\end{array}$} & \multicolumn{2}{|c|}{ GM (GSD) } & \multirow{2}{*}{$\begin{array}{l}\text { Max } \\
13.2\end{array}$} & \multicolumn{2}{|c|}{ GM (GSD) } & \multirow{2}{*}{$\begin{array}{l}\text { Max } \\
18.7\end{array}$} & \multicolumn{2}{|c|}{ GM (GSD) } & \multirow{2}{*}{$\begin{array}{l}\text { Max } \\
6.9\end{array}$} & \multirow{2}{*}{$\begin{array}{l}\text { ICC } \\
0.32\end{array}$} & \multicolumn{2}{|c|}{$(95 \% \mathrm{Cl})$} \\
\hline $\mathrm{BPA}^{\mathrm{b}}$ & 23.6 & 1.0 & $(2.5)$ & & 1.0 & $(2.4)$ & & 0.7 & $(2.30$ & & 0.89 & $(2.0$ & & & $(0.24$ & $0.41)$ \\
\hline MEHP & 8.0 & 4.9 & $(2.8)$ & 125 & 4.7 & $(2.9)$ & 152 & 5.0 & $(2.8)$ & 215 & 5.0 & $(2.2)$ & 215 & 0.25 & (0.17, & $0.35)$ \\
\hline MEHHP & 0 & 16.6 & (3.1) & 158 & 17.4 & $(3.2)$ & 1290 & 19.6 & $(2.9)$ & 383 & 18.6 & $(2.3)$ & 383 & 0.23 & (0.15, & $0.33)$ \\
\hline MEOHP & 0 & 8.9 & (3.1) & 85.0 & 10.4 & (3.2) & 730 & 11.8 & (2.9) & 244 & 10.8 & $(2.3)$ & 244 & 0.23 & $(0.15$ & $0.33)$ \\
\hline MECPP & 0.2 & 30.0 & $(2.7)$ & 252 & 31.9 & $(2.7)$ & 2650 & 31.9 & $(2.7)$ & 414 & 32.3 & $(2.1)$ & 414 & 0.26 & $(0.18$ & $0.36)$ \\
\hline$M B z \mathrm{P}^{\mathrm{b}}$ & 3.2 & 2.6 & $(4.0)$ & 69.6 & 2.4 & (3.6) & 38.5 & 4.2 & (2.6) & 109 & 3.2 & $(2.4)$ & 34.5 & 0.21 & $(0.14$ & $0.31)$ \\
\hline MBP & 0.3 & 59.0 & $(4.1)$ & 1340 & 49.0 & (3.8) & 1390 & 54.5 & (3.4) & 1190 & 56.0 & (2.6) & 468 & 0.28 & $(0.20$ & $0.38)$ \\
\hline $\mathrm{MiBP} \mathrm{P}^{\mathrm{b}}$ & 22.0 & 0.92 & $(4.7)$ & 118 & 0.7 & (4.6) & 26.5 & 1.9 & (2.9) & 40.1 & 1.2 & (2.9) & 32.4 & 0.31 & $(0.23$ & $0.40)$ \\
\hline MCPP & 8.0 & 1.1 & $(2.9)$ & 17.8 & 1.1 & $(2.8)$ & 15.0 & 1.1 & $(2.7)$ & 12.1 & 1.1 & $(2.2)$ & 6.6 & 0.31 & $(0.23$ & $0.40)$ \\
\hline MEP & 0.3 & 138 & $(4.2)$ & 4480 & 119 & (3.9) & 4980 & 112 & $(4.2)$ & 9810 & 126 & (3.1) & 2285 & 0.41 & (0.33, & $0.50)$ \\
\hline$\Sigma \mathrm{DEHP}$ & & 0.21 & $(2.7)$ & 1.7 & 0.22 & $(2.9)$ & 16.0 & 0.24 & $(2.7)$ & 4.3 & 0.23 & $(2.1)$ & 4.3 & 0.24 & (0.16, & $0.34)$ \\
\hline \multirow[t]{2}{*}{$\Sigma \mathrm{DBP}^{\mathrm{d}}$} & & 0.27 & $(4.1)$ & 6.1 & 0.23 & (3.7) & 6.3 & 0.26 & (3.3) & 5.4 & 0.26 & (2.6) & 2.2 & 0.28 & $(0.20$ & $0.38)$ \\
\hline & & \multicolumn{3}{|c|}{ Visit $1(n=92)$} & \multicolumn{3}{|c|}{ Visit $2(n=91)$} & \multicolumn{3}{|c|}{ Visit $3(n=108)$} & \multicolumn{3}{|c|}{ Prenatal GM $(n=109)$} & \multicolumn{3}{|c|}{ Variability } \\
\hline Males & $\%<\mathrm{LOD}$ & \multicolumn{2}{|c|}{ GM (GSD) } & Max & \multicolumn{2}{|c|}{ GM (GSD) } & Max & \multicolumn{2}{|c|}{ GM (GSD) } & Max & \multicolumn{2}{|c|}{ GM (GSD) } & Max & ICC & $(95 \% \mathrm{Cl}$ & \\
\hline $\mathrm{BPA}^{\mathrm{b}}$ & 26.8 & 0.93 & $(2.5)$ & 7.9 & 0.87 & $(2.3)$ & 9.2 & 0.64 & $(2.3)$ & 9.0 & 0.81 & (1.9) & 4.5 & 0.28 & (0.17, & 0.42) \\
\hline MEHP & 7.9 & 4.8 & $(2.6)$ & 53.0 & 4.4 & $(2.8)$ & 50.3 & 5.0 & $(2.9)$ & 215 & 5.0 & $(2.2)$ & 215 & 0.28 & (0.17, & $0.43)$ \\
\hline MEHHP & 0 & 15.6 & (3.4) & 150 & 15.7 & (3.0) & 85.0 & 19.9 & (2.9) & 383 & 18.0 & $(2.3)$ & 383 & 0.15 & (0.06, & $0.33)$ \\
\hline MEOHP ${ }^{b}$ & 0 & 8.3 & (3.3) & 80.2 & 9.4 & (3.0) & 53.5 & 12.0 & (2.8) & 244 & 10.4 & $(2.3)$ & 244 & 0.15 & (0.06, & $0.33)$ \\
\hline MECPP & 0.3 & 28.5 & (2.9) & 227 & 29.4 & $(2.5)$ & 134 & 32.7 & $(2.5)$ & 414 & 31.5 & $(2.1)$ & 414 & 0.15 & $(0.05$ & 0.33) \\
\hline$M B z P^{b}$ & 3.4 & 2.4 & (4.3) & 51.3 & 2.5 & (3.8) & 34.5 & 4.3 & (2.5) & 32.5 & 3.3 & (2.5) & 34.5 & 0.15 & (0.06, & 0.33) \\
\hline $\mathrm{MBP}$ & 0.7 & 54.1 & $(4.2)$ & 870 & 42.3 & (3.7) & 655 & 53.9 & (3.1) & 1000 & 53.0 & (2.6) & 468 & 0.24 & $(0.14$ & $0.40)$ \\
\hline $\mathrm{MiBP} \mathrm{P}^{\mathrm{b}}$ & 25.1 & 0.76 & (4.7) & 118 & 0.62 & (4.5) & 25.8 & 1.7 & (2.8) & 40.1 & 1.1 & (2.9) & 32.4 & 0.32 & (0.21, & $0.46)$ \\
\hline MCPP & 7.2 & 1.1 & $(2.7)$ & 9.8 & 1.1 & $(2.7)$ & 8.2 & 1.1 & (2.6) & 12.1 & 1.1 & (2.1) & 6.0 & 0.27 & (0.16, & $0.41)$ \\
\hline MEP & 0.3 & 128 & $(4.2)$ & 3260 & 117 & (3.6) & 2160 & 110 & (3.7) & 7950 & 124 & (3.0) & 2285 & 0.47 & (0.36, & $0.60)$ \\
\hline$\Sigma \mathrm{DEHP}$ & & 0.20 & (2.9) & 1.6 & 0.20 & (2.7) & 0.93 & 0.24 & (2.6) & 4.3 & 0.22 & (2.1) & 4.3 & 0.14 & $(0.05$ & $0.32)$ \\
\hline$\Sigma \mathrm{DBP}^{\mathrm{d}}$ & & 0.25 & $(4.1)$ & 4.5 & 0.20 & (3.7) & 3.0 & 0.25 & (3.1) & 4.5 & 0.25 & (2.6) & 2.1 & 0.25 & (0.15, & $0.40)$ \\
\hline
\end{tabular}

LOD limit of detection, GM geometric mean of samples collected during pregnancy, GSD geometric standard deviation, ICC intraclass correlation coefficient, $\mathrm{Cl}$ confidence interval

${ }^{\mathrm{a}} \mathrm{LODs}$ : BPA $=0.4, \mathrm{MEHP}, \mathrm{MEP}=1.0, \mathrm{MEHHP}, \mathrm{MEOHP}=0.1, \mathrm{MECPP}, \mathrm{MBz}, \mathrm{MiBP}, \mathrm{MCPP}=0.2, \mathrm{MBP}=0.5$; ${ }^{\mathrm{b}}$ Concentrations were significantly different across prenatal visits $(p<0.05) ;{ }^{c} \Sigma D E H P=$ molecular sum of MEHP, MEHHP, MEOHP, and MECPP $(\mu \mathrm{mol} / \mathrm{L}) ;{ }^{d} \Sigma \mathrm{DBP}=\mathrm{molar}$ sum of MnBP and MiBP $(\mu \mathrm{mol} / \mathrm{L})$ 
Table 2 Percent difference in peripubertal hormone levels associated with an IQR increase in phthalate metabolite or BPA concentration during trimester-specific in utero development ${ }^{\mathrm{a}}$

\begin{tabular}{|c|c|c|c|c|}
\hline & Visit $1(n=91)$ & Visit $2(n=90)$ & Visit $3(n=107)$ & Prenatal GM $(n=108)$ \\
\hline & $\% \Delta / \mathrm{IQR}(95 \% \mathrm{Cl})$ & $\% \Delta / \mathrm{IQR}(95 \% \mathrm{Cl})$ & $\% \Delta / \mathrm{IQR}(95 \% \mathrm{Cl})$ & $\% \Delta / \mathrm{IQR}(95 \% \mathrm{Cl})$ \\
\hline \multicolumn{5}{|l|}{ Estradiol } \\
\hline BPA & $-1.1(-10.8,9.7)$ & $-4.5(-14.2,6.2)$ & $0.1(-9.1,10.2)$ & $-2.4(-13.7,10.4)$ \\
\hline MBzP & $5.0(-3.5,14.3)$ & $0.7(-9.2,11.8)$ & $-2.0(-11.7,8.8)$ & $3.6(-7.4,16.0)$ \\
\hline MCPP & $5.3(-6.2,18.3)$ & $3.4(-9.8,18.6)$ & $3.7(-5.7,14.1)$ & $6.2(-7.4,21.8)$ \\
\hline MEP & $3.0(-7.9,15.2)$ & $-15.0(-25.1,-3.6)^{*}$ & $-0.6(-10.2,10.2)$ & $-3.4(-14.6,9.4)$ \\
\hline$\Sigma \mathrm{DEHP}$ & $11.2(1.5,21.9)^{*}$ & $8.2(-3.2,21.0)$ & $4.6(-4.8,14.9)$ & $11.2(-1.4,25.3)$ \\
\hline$\Sigma \mathrm{DBP}$ & $8.5(-0.7,18.5)$ & $-5.8(-15.4,4.8)$ & $7.6(-2.6,18.7)$ & $6.5(-5.5,20.1)$ \\
\hline \multicolumn{5}{|c|}{ Testosterone } \\
\hline BPA & $9.0(-16.6,42.3)$ & $14.8(-12.3,50.4)$ & $10.1(-16.7,45.5)$ & $33.4(-6.1,89.4)$ \\
\hline $\mathrm{MBzP}$ & $17.1(-5.9,45.7)$ & $-2.5(-25.1,27.0)$ & $3.1(-23.9,39.7)$ & $14.6(-17.1,58.5)$ \\
\hline MCPP & $9.9(-18.8,48.6)$ & $14.8(-18.8,62.4)$ & $9.5(-17.1,44.6)$ & $27.2(-14.2,88.7)$ \\
\hline MEP & $30.3(-2.0,73.2)$ & $-2.8(-30.3,35.6)$ & $-8.2(-31.8,23.5)$ & $6.6(-25.4,52.5)$ \\
\hline$\Sigma \mathrm{DEHP}$ & $10.8(-13.1,41.4)$ & $2.5(-23.1,36.5)$ & $8.4(-17.6,42.7)$ & $20.1(-15.2,70.1)$ \\
\hline$\Sigma \mathrm{DBP}$ & $4.8(-17.0,32.4)$ & $-2.9(-26.1,27.7)$ & $-9.3(-32.1,21.2)$ & $-0.3(-29.6,41.2)$ \\
\hline \multicolumn{5}{|l|}{ SHBG } \\
\hline BPA & $-0.8(-11.1,10.6)$ & $4.7(-6.2,16.9)$ & $5.3(-5.0,16.8)$ & $5.6(-7.4,20.5)$ \\
\hline $\mathrm{MBz} \mathrm{P}$ & $5.1(-3.9,14.9)$ & $-1.2(-11.3,10.0)$ & $15.2(3.2,28.5)^{*}$ & $10.2(-2.2,24.2)$ \\
\hline MCPP & $4.6(-7.4,18.3)$ & $-2.3(-15.2,12.5)$ & $14.4(3.5,26.5)^{*}$ & $12.5(-2.8,30.2)$ \\
\hline MEP & $3.4(-8.1,16.3)$ & $-2.8(-15.0,11.3)$ & $4.6(-6.4,16.8)$ & $4.2(-8.8,19.0)$ \\
\hline$\Sigma \mathrm{DEHP}$ & $-0.2(-9.7,10.2)$ & $1.9(-9.3,14.4)$ & $8.4(-2.1,19.9)$ & $8.2(-4.9,23.1)$ \\
\hline$\Sigma \mathrm{DBP}$ & $1.1(-8.0,11.2)$ & $-6.5(-16.2,4.4)$ & $12.3(1.1,24.9)^{*}$ & $5.2(-7.6,19.7)$ \\
\hline \multicolumn{5}{|l|}{ DHEA-S } \\
\hline BPA & $3.5(-10.8,20.0)$ & $-12.1(-23.9,1.6)$ & $1.2(-12.4,16.9)$ & $-1.9(-18.4,18.0)$ \\
\hline $\mathrm{MBzP}$ & $-0.9(-12.3,12.1)$ & $-15.9(-26.8,-3.4)^{*}$ & $-4.4(-18.3,11.8)$ & $-11.9(-25.5,4.0)$ \\
\hline MCPP & $-2.3(-17.4,15.5)$ & $-7.4(-23.3,11.8)$ & $-5.0(-17.6,9.7)$ & $-6.0(-23.5,15.4)$ \\
\hline MEP & $-4.9(-19.0,11.7)$ & $-9.2(-24.1,8.6)$ & $-7.7(-20.8,7.5)$ & $-9.4(-24.7,8.9)$ \\
\hline$\Sigma \mathrm{DEHP}$ & $0.9(-11.9,15.5)$ & $-4.4(-18.2,11.7)$ & $-0.1(-13.3,15.1)$ & $0.5(-16.2,20.5)$ \\
\hline$\Sigma \mathrm{DBP}$ & $-1.1(-13.1,12.6)$ & $-7.3(-20.0,7.5)$ & $-13.5(-25.3,0.2)$ & $-10.8(-25.4,6.7)$ \\
\hline \multicolumn{5}{|l|}{ Inhibin B } \\
\hline BPA & $3.0(-7.7,14.8)$ & $11.8(0.4,24.6)^{*}$ & $-1.8(-12.0,9.6)$ & $8.8(-5.2,24.9)$ \\
\hline $\mathrm{MBzP}$ & $2.7(-6.1,12.4)$ & $-2.9(-12.8,8.1)$ & $-6.5(-17.0,5.3)$ & $-5.6(-16.8,7.2)$ \\
\hline MCPP & $4.2(-7.8,17.8)$ & $8.4(-5.9,24.7)$ & $-0.9(-11.2,10.6)$ & $4.9(-10.2,22.5)$ \\
\hline MEP & $-1.6(-12.6,10.7)$ & $-1.3(-13.9,13.0)$ & $-13.6(-22.9,-3.2)^{*}$ & $-8.8(-20.6,4.9)$ \\
\hline ¿DEHP & $8.1(-2.0,19.3)$ & $7.0(-4.7,20.2)$ & $-2.9(-12.8,8.2)$ & $5.1(-8.3,20.5)$ \\
\hline$\Sigma \mathrm{DBP}$ & $2.9(-6.4,13.2)$ & $8.3(-3.0,21.0)$ & $-4.1(-14.4,7.5)$ & $2.3(-10.7,17.2)$ \\
\hline
\end{tabular}

*p $<0.05$

${ }^{a}$ Adjusted for child age, BMI z-score, and urinary specific gravity

with higher inhibin B levels, and MBzP, MCPP, and $\Sigma \mathrm{DBP}$ at prenatal visit 3 were all associated with higher SHBG levels. Geometric mean levels of phthalate metabolite and BPA concentrations across pregnancy were not significantly associated with peripubertal serum hormone concentrations (Table 2).
Although logistic regression models including SG as a separate covariate (Additional file 1: Table S3) yielded results similar to models including SG-corrected phthalate metabolite and BPA values, the latter generally provided narrower confidence intervals. Therefore, we have presented ORs from models with SG-corrected exposure 
measures in Table 3. With the exception of MEP at visit 1 , maternal phthalate metabolite and BPA levels at prenatal visits 1 and 3, as well as the GM of concentrations across pregnancy, were all associated with reduced odds of adrenarche $(\mathrm{PH}>1)$ after adjustment for child age and BMI z-score (Table 3). However, MBzP and $\Sigma D E H P$ at visit 3 , and the $\mathrm{GM}$ of $\mathrm{MBzP}$ across pregnancy were the only associations to reach statistical significance. Pubertal onset (GD $>1$ or TV $>3 \mathrm{~mL}$ ) was not associated with prenatal phthalate metabolite and BPA concentrations measured at any study visit or with GM concentrations across pregnancy. The one exception to this was reduced odds of TV $>3 \mathrm{~mL}$ associated with higher GM MBzP concentrations.

Population means (fixed effects) and distributions of individual exposure intercepts and slopes (random effects) from mixed models evaluating the effects of gestational age on prenatal phthalate and BPA levels are presented in Additional file 1: Table S4. When we considered individual's exposure intercept and slope as predictors of peripubertal hormone levels we observed relationships that were consistent with what we observed using the visit specific concentrations. For instance, an IQR increase in the
$\Sigma$ DEHP intercept, which represents exposure in the first trimester, was associated with 15\% higher peripubertal estradiol concentrations (Table 4). This is consistent with our main analyses, in which we observed $11 \%$ higher estradiol with an IQR increase $\Sigma$ DEHP at visit 1 (Table 2). Further, an IQR increase in MEP slope, which represents the change in exposure across pregnancy, was associated with 13\% higher inhibin B (Table 4), while in our main analysis an IQR increase in MEP levels at prenatal visit 3 was significantly associated with $14 \%$ higher inhibin B (Table 2). Consistent with results from our main analysis in Table 3, estimated exposure at 7 weeks (exposure intercepts) were not significantly associated with odds of puberty or adrenarche $(\mathrm{GD}>1, \mathrm{TV}>3$, or $\mathrm{PH}>1$ ) (Table 5 ), although estimated relationships were in similar directions.

\section{Discussion}

We investigated maternal urinary phthalate metabolite and BPA concentrations during the first, second, and third trimesters of pregnancy in relation to peripubertal reproductive hormone levels and measures of sexual maturation in male offspring in order to identify potential windows of exposure susceptibility during in utero

Table 3 Odds of Tanner stage $>1$ or TV $>3 \mathrm{ml}$ associated with an IQR increase in trimester-specific, SG-corrected in utero urinary phthalate metabolite or BPA concentration ${ }^{a}$

\begin{tabular}{|c|c|c|c|c|}
\hline & Visit $1(n=90)$ & Visit $2(n=89)$ & Visit $3(n=105)$ & Prenatal GM $(n=106)$ \\
\hline & OR/IQR (95\% Cl) & OR/IQR (95\% Cl) & OR/IQR (95\% Cl) & OR/IQR (95\% Cl) \\
\hline \multicolumn{5}{|c|}{ Genital Development } \\
\hline BPA & $0.71(0.39,1.28)$ & $1.41(0.78,2.54)$ & $0.93(0.53,1.61)$ & $1.04(0.5,2.14)$ \\
\hline $\mathrm{MBzP}$ & $0.95(0.58,1.55)$ & $0.98(0.57,1.68)$ & $0.71(0.37,1.36)$ & $0.75(0.37,1.51)$ \\
\hline MCPP & $1.2(0.61,2.36)$ & $1.16(0.53,2.54)$ & $0.97(0.53,1.77)$ & $1.14(0.48,2.73)$ \\
\hline MEP & $1.5(0.79,2.85)$ & $1.17(0.56,2.48)$ & $1.09(0.58,2.03)$ & $1.27(0.58,2.81)$ \\
\hline$\Sigma D E H P$ & $0.99(0.56,1.74)$ & $0.85(0.46,1.57)$ & $0.71(0.4,1.29)$ & $0.77(0.37,1.62)$ \\
\hline$\Sigma \mathrm{DBP}$ & $0.77(0.47,1.27)$ & $0.86(0.48,1.52)$ & $0.59(0.31,1.13)$ & $0.57(0.27,1.21)$ \\
\hline \multicolumn{5}{|l|}{ Pubic Hair } \\
\hline BPA & $0.2(0.02,2.76)$ & $1.1(0.31,3.9)$ & $0.42(0.13,1.36)$ & $0.72(0.19,2.73)$ \\
\hline $\mathrm{MBz} \mathrm{P}$ & $0.13(0.01,1.56)$ & $0.99(0.26,3.73)$ & $0.18(0.03,0.97)^{*}$ & $0.31(0.1,0.93)^{*}$ \\
\hline MCPP & $0.33(0.02,4.72)$ & $5.99(0.5,72.02)$ & $0.47(0.16,1.37)$ & $0.5(0.11,2.26)$ \\
\hline MEP & $2.04(0.36,11.58)$ & $1.31(0.22,7.85)$ & $0.44(0.15,1.24)$ & $0.52(0.17,1.6)$ \\
\hline$\Sigma \mathrm{DEHP}$ & $0(0,542.1)$ & $2.22(0.28,17.34)$ & $0.27(0.07,0.99)^{*}$ & $0.16(0.03,1.02)$ \\
\hline$\Sigma \mathrm{DBP}$ & $0.28(0.02,3.85)$ & $2.68(0.48,15.08)$ & $0.36(0.13,1.06)$ & $0.39(0.11,1.42)$ \\
\hline \multicolumn{5}{|c|}{ Testicular Volume } \\
\hline BPA & $1.28(0.67,2.45)$ & $2.01(0.94,4.28)$ & $1.34(0.68,2.66)$ & $2.29(0.87,5.98)$ \\
\hline $\mathrm{MBz} \mathrm{P}$ & $0.7(0.39,1.23)$ & $0.62(0.3,1.29)$ & $0.73(0.33,1.6)$ & $0.4(0.16,1)^{*}$ \\
\hline MCPP & $0.56(0.25,1.27)$ & $1.59(0.6,4.23)$ & $1.03(0.5,2.12)$ & $1.07(0.36,3.19)$ \\
\hline MEP & $1.33(0.64,2.76)$ & $0.75(0.31,1.86)$ & $1.32(0.6,2.91)$ & $1.16(0.44,3.06)$ \\
\hline$\Sigma D E H P$ & $1.21(0.64,2.3)$ & $0.99(0.49,2)$ & $1.5(0.75,3)$ & $1.94(0.7,5.34)$ \\
\hline$\Sigma \mathrm{DBP}$ & $1(0.54,1.84)$ & $1.66(0.85,3.25)$ & $0.88(0.44,1.75)$ & $1.35(0.54,3.4)$ \\
\hline
\end{tabular}

${ }^{*} p<0.05$

${ }^{\mathrm{a} A d j u s t e d}$ for child age and BMI z-score 
Table 4 Relationships between IQR increases in estimated intercepts and slopes of exposure across in utero development and hormone levels at $8-14$ years $(n=104)^{a}$

\begin{tabular}{|c|c|c|c|c|c|c|}
\hline & & Estradiol & Testosterone & SHBG & DHEA-S & Inhibin B \\
\hline & & $\% \Delta / \mathrm{IQR}(95 \% \mathrm{Cl})$ & $\% \Delta / \mathrm{IQR}(95 \% \mathrm{Cl})$ & $\% \Delta / \mathrm{IQR}(95 \% \mathrm{Cl})$ & $\% \Delta / \mathrm{IQR}(95 \% \mathrm{Cl})$ & $\% \Delta / \mathrm{IQR}(95 \% \mathrm{Cl})$ \\
\hline$\overline{\mathrm{BPA}^{\mathrm{b}}}$ & Intercept & $-3.6(-13.6,7.5)$ & $12.8(-16,51.6)$ & $2.3(-8.8,14.9)$ & $-0.9(-15.1,15.7)$ & $5.7(-6.1,18.9)$ \\
\hline & Slope & $\mathrm{n} / \mathrm{a}$ & $n / a$ & $\mathrm{n} / \mathrm{a}$ & $\mathrm{n} / \mathrm{a}$ & $\mathrm{n} / \mathrm{a}$ \\
\hline $\mathrm{MBz} \mathrm{P}^{\mathrm{b}}$ & Intercept & $6.1(-4.5,17.9)$ & $20.4(-9.5,60.1)$ & $9.8(-1.7,22.6)$ & $-5.4(-18.5,9.9)$ & $1.5(-9.6,13.8)$ \\
\hline & Slope & $\mathrm{n} / \mathrm{a}$ & $\mathrm{n} / \mathrm{a}$ & $\mathrm{n} / \mathrm{a}$ & $\mathrm{n} / \mathrm{a}$ & $\mathrm{n} / \mathrm{a}$ \\
\hline MCPP & Intercept & $8.3(-5.3,24.0)$ & $9.4(-24.2,57.8)$ & $15.3(0.3,32.6)^{*}$ & $-7.1(-23.2,12.5)$ & $5.6(-8.7,22.3)$ \\
\hline & Slope & $2.7(-9.7,16.9)$ & $-0.5(-30.0,41.3)$ & $14.5(0.2,30.9)^{*}$ & $-7.3(-22.8,11.4)$ & $-1.4(-14.3,13.3)$ \\
\hline MEP & Intercept & $-6.3(-17.2,6.1)$ & $6.7(-23.5,48.8)$ & $4.9(-8.1,19.7)$ & $-6.7(-21.8,11.3)$ & $-6.8(-18.3,6.4)$ \\
\hline & Slope & $-7.4(-16.8,3.1)$ & $-22.1(-41.6,3.8)$ & $0.0(-10.8,12.1)$ & $-4.8(-18.3,10.9)$ & $-12.9(-22.3,-2.4)^{*}$ \\
\hline$\Sigma \mathrm{DEHP}$ & Intercept & $15.0(3.2,28.2)^{*}$ & $6.2(-21.6,43.9)$ & $9.4(-2.8,23.0)$ & $0.4(-14.4,17.7)$ & $6.6(-5.4,20.2)$ \\
\hline & Slope & $0.9(-9.7,12.8)$ & $-9.1(-33.3,24.0)$ & $9.2(-3.2,23.0)$ & $-2.2(-16.8,15.1)$ & $-6.6(-17.3,5.6)$ \\
\hline$\Sigma \mathrm{DBP}$ & Intercept & $8.7(-7.0,27.1)$ & $-12.4(-42.6,33.7)$ & $11.2(-5.7,31.1)$ & $-14.9(-31.7,5.9)$ & $5.1(-11.3,24.5)$ \\
\hline & Slope & $0.7(-12.1,15.2)$ & $-19.8(-44.4,15.7)$ & $10.5(-4.2,27.4)$ & $-14.4(-29.2,3.6)$ & $-2.7(-15.9,12.7)$ \\
\hline
\end{tabular}

${ }^{*} p<0.05$

${ }^{a}$ Adjusted for child age and BMI z-score. ${ }^{b}$ Intercept and slope highly correlated (BPA $r=-0.82$, MBzP $r=-0.99$ ), only intercept included in model

development. Our findings suggest that compared to phthalate exposure early in pregnancy, exposure specifically during the third trimester is associated with reduced odds of adrenarche and a corresponding increase in serum SHBG concentrations. In addition, we observed an increase in peripubertal serum estradiol associated with DEHP exposure specifically in the first trimester of in utero development.

In comparison with our previous evaluation of third trimester phthalate levels and sexual maturation in males in this same population [17], estimated associations for first and second trimester exposure also indicated reduced odds of adrenarche $(\mathrm{PH}>1)$ with phthalate exposure, although associations did not reach statistical significance. Consistent with reduced odds of adrenarche, we observed reduced peripubertal serum DHEA-S concentrations in relation to several phthalate metabolite levels across

Table 5 Relationships between an IQR increase in estimated exposure at 7 weeks gestation (intercepts) and odds of Tanner stage $>1$ or $\mathrm{TV}>3 \mathrm{~mL}(n=102)^{\mathrm{a}}$

\begin{tabular}{clll}
\hline & $\begin{array}{l}\text { Genital Development } \\
\text { OR/IQR }(95 \% \mathrm{Cl})\end{array}$ & $\begin{array}{l}\text { Pubic Hair } \\
\text { OR/IQR }(95 \% \mathrm{Cl})\end{array}$ & $\begin{array}{l}\text { Testicular Volume } \\
\text { OR/IQR }(95 \% \mathrm{Cl})\end{array}$ \\
\hline Intercept & & & \\
BPA & $0.86(0.46,1.58)$ & $0.76(0.20,2.98)$ & $1.67(0.79,3.56)$ \\
MBzP & $0.82(0.44,1.52)$ & $0.54(0.18,1.62)$ & $0.55(0.25,1.22)$ \\
MCPP & $1.17(0.64,2.15)$ & $1.33(0.43,4.15)$ & $0.66(0.31,1.43)$ \\
MEP & $1.33(0.67,2.61)$ & $1.08(0.33,3.54)$ & $1.26(0.56,2.86)$ \\
¿DEHP & $0.82(0.46,1.45)$ & $0.41(<0.001,1.46)$ & $1.29(0.65,2.56)$ \\
¿DBP & $0.65(0.34,1.23)$ & $0.94(0.29,3.09)$ & $1.29(0.56,2.96)$ \\
\hline
\end{tabular}

${ }^{\mathrm{a}}$ Adjusted for child age and BMI $\mathrm{z}$-score pregnancy, although only $\mathrm{MBzP}$ in the second trimester was statistically significant (Table 2).

Two prior studies examined associations between markers of in utero phthalate exposure and reproductive hormone concentrations in cord blood, one of which reported no relationship between third trimester maternal urinary phthalate levels and hormones among male newborns [27]. However, the second study reported associations between third trimester MEHP levels in maternal blood and reduced testosterone to estradiol ratios and inhibin $\mathrm{B}$ among males [10]. In addition, a cross-sectional study of perinatal phthalate exposure reported that MEP and MBP concentrations in breast milk were associated with increased luteinizing hormone to free testosterone ratios and SHBG in male infants at three months of age [28]. Findings from the latter two studies, as well as the associations between several phthalate metabolite levels during the third trimester and higher peripubertal SHBG concentrations reported in the present study, are consistent with anti-androgen exposure.

A number of studies have examined associations between in utero phthalate exposure and fetal reproductive tract development in males. One of the first studies of phthalate exposure found that maternal urinary MEP, MBP, MBzP, and MiBP levels during the third trimester were associated with shortened AGD in male infants [12]. In a recent study, researchers found that maternal urinary DEHP metabolite concentrations in the first trimester, but not the second and third trimesters, were inversely associated with this same outcome measure [13]. This is consistent with what is currently known about male reproductive development, as AGD is determined by androgen action during the masculinizing programming 
window, which in humans is thought to be between weeks 7-15 of in utero development [19]. Interestingly, we observed a similar relationship between DEHP metabolites in the first trimester and increased serum estradiol levels in males at 8-14 years of age, but saw no association with exposure during later trimesters. However, we did not observe associations between in utero phthalate exposure and peripubertal testosterone levels, as we might expect given phthalates anti-androgenic activity.

One previous study reported positive correlations between cord blood levels of BPA with inhibin B and testosterone among male infants [11], which although measured in different media and at different time points, is consistent with our finding of increased peripubertal inhibin $B$ concentrations in relation to urinary BPA at prenatal visit 2. However, in the current study, prenatal BPA levels were not associated with any other hormone or measure of sexual maturation among boys at 8-14 years of age.

Although mechanisms by which in utero phthalate exposure may alter fetal and pubertal reproductive development are unclear, animal studies have shown that prenatal phthalate exposure may interfere with differentiation and function of both Sertoli and Leydig cells, as well as testosterone production in the testes [29-35], possibly by disrupting the hypothalamus-pituitary-gonadal axis and/or via induction of oxidative stress [36-38]. Steroid hormone levels are important in all phases of fetal male reproductive system development, which begins at gestational week 6 with the differentiation of gonadal tissue into fetal testes and is mostly complete by week 15 , although growth and testicular descent continue through the third trimester. Considering the complexity of this process throughout the gestational period, there is substantial potential for differential windows of susceptibility to exposure during this time. Indeed, both animal and human studies have demonstrated that exposure to androgens, anti-androgens, or estrogens during different periods of in utero development have different effects on the newborn male reproductive tract $[13,18,19,39,40]$. To our knowledge, the current study is the first to investigate the long term impact of phthalate and BPA exposure during specific periods of in utero development on male reproductive development during puberty.

Our analyses examining exposure levels and rates of change across pregnancy in relation to reproductive hormones and sexual maturation enabled us to look at exposure across time within the same mother/child pair rather than at discrete time points. Our findings using this method were consistent with those from our main analysis, thus our conclusions are robust to the method of analysis used.

Our analysis was limited by a relatively small sample size, and we made a large number of comparisons, increasing the likelihood of chance findings. Also, few boys had a PH Tanner stage $>1$, which may have resulted in unstable effect estimates (i.e. large confidence intervals). However, we are currently conducting an additional follow-up of over 550 children in the ELEMENT cohort, including boys in the current analysis who are now 917 years of age, for repeated hormone measurements and assessments of sexual maturation. Timing of the prenatal period may also have influenced our findings, as decreases in exposure to some phthalates have been demonstrated in the US since the early 2000s [41]. Because our study included a range of ages (8-14 years), older boys may have been more likely to have higher in utero phthalate exposure as well as to have begun puberty. However, it is not known if these same exposure trends occurred in Mexico during the time of mother's enrollment in this study (1997-2004), and when we additionally adjusted our models for year of conception, our results did not materially change. We also measured serum hormones using standardized immunoassays rather than the preferred method of LC/MS-MS, although hormone concentrations were all above their respective LODs, with the exception of testosterone in 12 samples. In addition, hormone concentrations were measured at only one time point and not adjusted for time of day, leading to potential non-differential misclassification due to diurnal variation. Another limitation was measurements of urinary phthalate metabolites at only one time point during each trimester, which may not fully characterize exposure across in utero development.

\section{Conclusions}

Our results suggest that associations between in utero phthalate and BPA exposure and peripubertal measures of male reproductive development are dependent on the timing of that exposure during gestation. These findings are consistent with current understanding of male reproductive tract development during the fetal and pubertal periods, and with reports from animal research. Although our findings need to be repeated in larger and more mature populations, future epidemiological studies of in utero exposure to endocrine disrupting chemicals and reproductive development should consider potential windows of susceptibility.

\section{Additional file}

Additional file 1: Table S1. Spearman Correlations between trimester specific urinary specific gravity and phthalate metabolites or BPA measurements from that trimester. Table $\mathbf{S 2}$. Number of boys included in trimester-specific analyses who had Tanner Stage $=1$ vs $>1$, and testicular volume $\leq 3 \mathrm{ml}$ vs $>3 \mathrm{ml}$. Table $\mathbf{S 3}$. Odds of Tanner stage $>1$ or TV $>3 \mathrm{ml}$ associated with an IQR increase in uncorrected in utero urinary phthalate metabolite or BPA concentration. Table S4. Fixed and random effect estimates of gestational age on In-transformed, SG-standardized phthalate metabolite and BPA concentrations in prenatal urine samples. (DOCX 29 kb) 


\section{Abbreviations}

AGD: Anogenital distance; BMI: Body mass index; BPA: Bisphenol A; Cl: Confidence interval; DBP: Dibutyl phthalate; DEHP: Di-2-ethylhexyl phthalate; DHEA-S: Dehydroepiandrosterone sulfate; EDC: Endocrine disrupting chemical; ELEMENT: Early Life Exposure in Mexico to Environmental Toxicants; GD: Tanner genital stage; GM: Geometric mean; ICC: Intraclass correlation coefficient; ID-LC-MS/MS: Isotope dilution-liquid chromatography-tandem mass spectrometry; IQR: Interquartile range; LOD: Limit of detection; MBzP: Monobenzyl phthalate; MCPP: Mono-3carboxypropyl phthalate; MECPP: Mono-2-ethyl-5-carboxypentyl phthalate; MEHHP: Mono-2-ethyl-5-hydroxyhexyl phthalate; MEHP: Mono-2-ethylhexyl phthalate; MEOHP: Mono-2-ethyl-5-oxohexyl phthalate; MEP: Monoethyl phthalate; MiBP: Monoisobutyl phthalate; MnBP: Mono- $n$-butyl phthalate; OR: Odds ratio; PH: Tanner pubic hair stage; SG: Specific gravity; SHBG: Sex hormone-binding globulin; TV: Testicular volume

\section{Acknowledgements}

We would like to thank $A B C$ Hospital for providing facilities for this research.

\section{Funding}

This work was supported by grants P01ES022844 from the National Institute for Environmental Health Sciences (NIEHS), and RD 83543601 from the US Environmental Protection Agency (US EPA). Its contents are solely the responsibility of the grantee and do not necessarily represent the official views of the US EPA. Further, the US EPA does not endorse the purchase of any commercial products or services mentioned in the publication. This work was also supported and partially funded by the National Institute of Public Health, Ministry of Health of Mexico.

\section{Availability of data and materials}

Please contact author for data requests.

\section{Authors' contributions}

DJW, analyzed and interpreted data, drafted and revised manuscript; BNS, conception of study and study design, guided data analysis and interpretation, revised manuscript; MMT-R., conception of study and study design, interpreted data, revised manuscript; JML, interpreted data, revised manuscript; AM-G, collection of data, revised manuscript; CB-G, collection of data, revised manuscript; KEP, conception of study and study design, interpreted data, revised manuscript; JDM, conception of study and study design, interpreted data, revised manuscript. All authors read and approved the final manuscript.

\section{Competing interests}

The authors declare that they have no competing interests.

\section{Consent for publication}

Not applicable.

\section{Ethics approval and consent to participate}

Research protocols were approved by the ethics and research committees of the Mexico National Institute of Public Health and the University of Michigan (HUM00034344), and all participants provided informed consent prior to enrollment.

\section{Publisher's Note}

Springer Nature remains neutral with regard to jurisdictional claims in published maps and institutional affiliations.

\section{Author details}

${ }^{1}$ Department of Environmental Health Sciences, University of Michigan School of Public Health, 6611C SPH I, 1415 Washington Heights, Ann Arbor, Ml 48109, USA. ²Department of Biostatistics, University of Michigan School of Public Health, Ann Arbor, MI, USA. ${ }^{3}$ Center for Nutrition and Health Research, Instituto Nacional de Salud Pública, MOR, Cuernavaca, MEXICO. ${ }^{4}$ Pediatric Endocrinology, Child Health Evaluation and Research Unit (CHEAR), University of Michigan, Ann Arbor, MI, USA. ${ }^{5}$ American British Cowdray (ABC) Hospital, Mexico City, MEXICO. ${ }^{6}$ Department of Nutritional Sciences, University of Michigan School of Public Health, Ann Arbor, MI, USA. ${ }^{7}$ Center for Human Growth and Development, University of Michigan, Ann Arbor, MI, USA.
Received: 23 November 2016 Accepted: 11 June 2017

Published online: 21 June 2017

\section{References}

1. Zoeller RT, Brown TR, Doan LL, Gore AC, Skakkebaek NE, Soto AM, et al. Endocrine-disrupting chemicals and public health protection: A statement of principles from The Endocrine Society. Endocrinology. 2012;153(9):4097-110.

2. Silva MJ, Barr DB, Reidy JA, Malek NA, Hodge CC, Caudill SP, et al. Urinary levels of seven phthalate metabolites in the US population from the National Health and Nutrition Examination Survey (NHANES) 1999-2000. Environ Health Perspect. 2004;112(3):331-8.

3. Teitelbaum SL, Britton JA, Calafat AM, Ye X, Silva MJ, Reidy JA, et al. Temporal variability in urinary concentrations of phthalate metabolites, phytoestrogens and phenols among minority children in the United States. Environ Res. 2008;106(2):257-69.

4. Calafat AM, Ye X, Wong LY, Reidy JA, Needham LL. Exposure of the U.S. population to bisphenol A and 4-tertiary-octylphenol: 2003-2004. Environ Health Perspect. 2008;116(1):39-44

5. Mok-Lin E, Ehrlich S, Williams PL, Petrozza J, Wright DL, Calafat AM, et al. Urinary bisphenol A concentrations and ovarian response among women undergoing IVF. Int J Androl. 2010;33(2):385-93.

6. Ehrlich S, Williams PL, Missmer SA, Flaws JA, Ye XY, Calafat AM, et al. Urinary bisphenol A concentrations and early reproductive health outcomes among women undergoing IVF. Hum Reprod. 2012;27(12):3583-92.

7. Meeker JD, Calafat AM, Hauser R. Urinary metabolites of di(2-ethylhexyl) phthalate are associated with decreased steroid hormone levels in adult men. J Androl. 2009:30(3):287-97.

8. Pan G, Hanaoka T, Yoshimura M, Zhang S, Wang P, Tsukino H, et al. Decreased serum free testosterone in workers exposed to high levels of di-n-butyl phthalate (DBP) and di-2-ethylhexyl phthalate (DEHP): a crosssectional study in China. Environ Health Perspect. 2006:114(11):1643-8.

9. Sathyanarayana S, Barrett E, Butts S, Wang C, Swan SH. Phthalate exposure and reproductive hormone concentrations in pregnancy. Reproduction. 2014;147(4):401-9.

10. Araki A, Mitsui T, Miyashita C, Nakajima T, Naito H, Ito S, et al. Association between maternal exposure to di(2-ethylhexyl) phthalate and reproductive hormone levels in fetal blood: the Hokkaido study on environment and children's health. PLoS One. 2014;9(10):e109039.

11. Fenichel P, Dechaux H, Harthe C, Gal J, Ferrari P, Pacini $P$, et al. Unconjugated bisphenol A cord blood levels in boys with descended or undescended testes. Hum Reprod. 2012;27(4):983-90.

12. Swan SH, Main KM, Liu F, Stewart SL, Kruse RL, Calafat AM, et al. Decrease in anogenital distance among male infants with prenatal phthalate exposure. Environ Health Perspect. 2005:113(8):1056-61.

13. Martino-Andrade AJ, Liu F, Sathyanarayana S, Barrett ES, Redmon JB, Nguyen $\mathrm{RH}$, et al. Timing of prenatal phthalate exposure in relation to genital endpoints in male newborns. Andrology. 2016;4:585-93.

14. Swan SH, Sathyanarayana S, Barrett ES, Janssen S, Liu F, Nguyen RH, et al. First trimester phthalate exposure and anogenital distance in newborns. Hum Reprod. 2015:30(4):963-72.

15. Eisenberg ML, Hsieh MH, Walters RC, Krasnow R, Lipshultz LI. The relationship between anogenital distance, fatherhood, and fertility in adult men. PLoS One. 2011;6(5):e18973.

16. Skakkebaek NE, Rajpert-De Meyts E, Buck Louis GM, Toppari J, Andersson AM, Eisenberg ML, et al. Male reproductive disorders and fertility trends: Influences of environment and genetic susceptibility. Physiol Rev. 2016; 96(1):55-97.

17. Ferguson KK, Peterson KE, Lee JM, Mercado-Garcia A, Goldenberg CB, Tellez-Rojo MM, et al. Prenatal and peripubertal phthalates and bisphenol $\mathrm{A}$ in relation to sex hormones and puberty in boys. Reprod Toxicol. 2014:47:70-6.

18. Macleod DJ, Sharpe RM, Welsh M, Fisken M, Scott HM, Hutchison GR, et al. Androgen action in the masculinization programming window and development of male reproductive organs. Int J Androl. 2010;33(2):279-87.

19. Welsh M, Saunders PT, Fisken M, Scott HM, Hutchison GR, Smith LB, et al. Identification in rats of a programming window for reproductive tract masculinization, disruption of which leads to hypospadias and cryptorchidism. J Clin Invest. 2008;118(4):1479-90.

20. Lewis RC, Meeker JD, Peterson KE, Lee JM, Pace GG, Cantoral A, et al. Predictors of urinary bisphenol $\mathrm{A}$ and phthalate metabolite concentrations in Mexican children. Chemosphere. 2013;93(10):2390-8. 
21. World Health Organization. Growth reference data for 5-19 years. 2007. http://www.who.int/growthref/en/. Accessed 15 Aug 2016.

22. Marshall WA, Tanner JM. Variations in pattern of pubertal changes in girls. Arch Dis Child. 1969:44(235):291-303.

23. Mouritsen A, Aksglaede L, Soerensen K, Hagen CP, Petersen JH, Main KM, et al. The pubertal transition in 179 healthy Danish children: associations between pubarche, adrenarche, gonadarche, and body composition. Eur J Endocrinol. 2013;168(2):129-36.

24. Spiegelman D. The SAS ICC9 Macro. 2016. https://www.hsph.harvard.edu/ donna-spiegelman/software/icc9/. Accessed 15 August 2016.

25. Peduzzi P, Concato J, Kemper E, Holford TR, Feinstein AR. A simulation study of the number of events per variable in logistic regression analysis. J Clin Epidemiol. 1996;49(12):1373-9.

26. Vittinghoff E, McCulloch CE. Relaxing the rule of ten events per variable in logistic and Cox regression. Am J Epidemiol. 2007:165(6):710-8.

27. Lin LC, Wang SL, Chang YC, Huang PC, Cheng JT, Su PH, et al. Associations between maternal phthalate exposure and cord sex hormones in human infants. Chemosphere. 2011;83(8):1192-9.

28. Main KM, Mortensen GK, Kaleva MM, Boisen KA, Damgaard IN, Chellakooty $\mathrm{M}$, et al. Human breast milk contamination with phthalates and alterations of endogenous reproductive hormones in infants three months of age. Environ Health Perspect. 2006:114(2):270-6.

29. Fisher JS, Macpherson S, Marchetti N, Sharpe RM. Human 'testicular dysgenesis syndrome': a possible model using in-utero exposure of the rat to dibutyl phthalate. Hum Reprod. 2003;18(7):1383-94.

30. Wang Y, Yang Q, Liu W, Yu M, Zhang Z, Cui X. Di(2-ethylhexyl) phthalate exposure in utero damages sertoli cell differentiation via disturbance of sex determination pathway in fetal and postnatal mice. Toxicol Sci. 2016;152:53-61.

31. Aydogan Ahbab M, Barlas N. Influence of in utero di-n-hexyl phthalate and dicyclohexyl phthalate on fetal testicular development in rats. Toxicol Lett. 2015;233(2):125-37.

32. Culty M, Thuillier R, Li W, Wang Y, Martinez-Arguelles DB, Benjamin CG, et al. In utero exposure to di-(2-ethylhexyl) phthalate exerts both short-term and long-lasting suppressive effects on testosterone production in the rat. Biol Reprod. 2008;78(6):1018-28.

33. Foster PM. Disruption of reproductive development in male rat offspring following in utero exposure to phthalate esters. Int J Androl. 2006;29(1):140-7.

34. Kumar N, Srivastava S, Roy P. Impact of low molecular weight phthalates in inducing reproductive malfunctions in male mice: Special emphasis on Sertoli cell functions. Gen Comp Endocrinol. 2015;215:36-50.

35. Shultz VD, Phillips S, Sar M, Foster PM, Gaido KW. Altered gene profiles in fetal rat testes after in utero exposure to di(n-butyl) phthalate. Toxicol Sci. 2001;64(2):233-42.

36. Ha M, Guan X, Wei L, Li P, Yang M, Liu C. Di-(2-ethylhexyl) phthalate inhibits testosterone level through disturbed hypothalamic-pituitary-testis axis and ERK-mediated 5alpha-Reductase 2. Sci Total Environ. 2016;563-564:566-75.

37. Sedha S, Kumar S, Shukla S. Role of oxidative stress in male reproductive dysfunctions with reference to phthalate compounds. Urol J. 2015;12(5):2304-16.

38. Sobarzo CM, Rosana Nde M, Livia L, Berta D, Schteingart HF. Mono-(2ethylhexyl) phthalate (MEHP) affects intercellular junctions of Sertoli cell: A potential role of oxidative stress. Reprod Toxicol. 2015;58:203-12.

39. Zheng Z, Armfield BA, Cohn MJ. Timing of androgen receptor disruption and estrogen exposure underlies a spectrum of congenital penile anomalies. Proc Natl Acad Sci U S A. 2015;112(52):E7194-203.

40. Nef S, Parada LF. Hormones in male sexual development. Genes Dev. 2000;14(24):3075-86.

41. Zota AR, Calafat AM, Woodruff TJ. Temporal trends in phthalate exposures: Findings from the national health and nutrition examination survey, 2001-2010. Environ Health Perspect. 2014;122:235-41.

\section{Submit your next manuscript to BioMed Central and we will help you at every step:}

- We accept pre-submission inquiries

- Our selector tool helps you to find the most relevant journal

- We provide round the clock customer support

- Convenient online submission

- Thorough peer review

- Inclusion in PubMed and all major indexing services

- Maximum visibility for your research

Submit your manuscript at www.biomedcentral.com/submit
Biomed Central 